\title{
Abnormally elevated ubiquilin-1 expression in breast cancer regulates metastasis and stemness via AKT signaling
}

\author{
XIAOYUE FENG ${ }^{1,2}$, ANNA CAO $^{3}$, TAO QIN $^{4}$, QINGQING ZHANG ${ }^{1}$, SHUJUN FAN $^{1}$, \\ BO WANG ${ }^{1}$, BO SONG ${ }^{1}$, XIAOTANG YU ${ }^{1}$ and LIANHONG LI ${ }^{1}$ \\ ${ }^{1}$ Department of Pathology and Forensic Medicine, College of Basic Medical Science, \\ Dalian Medical University, Dalian, Liaoning 116044; ${ }^{2}$ Department of Pathology, Zhejiang Hospital, \\ Hangzhou, Zhejiang 310013; ${ }^{3}$ Department of Pathology, Tongde Hospital of Zhejiang Province, Hangzhou, \\ Zhejiang 310000; ${ }^{4}$ Department of Oncology, Qingdao Municipal Hospital, Qingdao, Shandong 266071, P.R. China
}

Received March 30, 2021; Accepted August 10, 2021

DOI: $10.3892 / o r .2021 .8187$

\begin{abstract}
Ubiquilin-1 (UBQLN1) is an essential factor for the maintenance of proteostasis in cells. It is important for the regulation of different protein degradation mechanisms, including the ubiquitin-proteasome system, autophagy and endoplasmic reticulum-associated protein degradation pathways. However, the role of UBQLN1 in cancer progression remains largely unknown. In the present study, the expression, functions and molecular mechanisms of UBQLN1 in breast cancer tissue samples and cell lines were explored. Immunohistochemical and bioinformatics analyses revealed that UBQLN1 expression was significantly upregulated in breast cancer tissues and cell lines. UBQLN1 expression in breast cancer was significantly associated with lymph node metastasis and TNM stage. Moreover, a high UBQLN1 expression was a predictor of an unfavorable survival in patients with breast cancer. In vitro, UBQLN1 silencing markedly inhibited cell migration and invasion, epithelial-to-mesenchymal transition (EMT) and MMP expression. UBQLN1 silencing attenuated the stem cell-like properties of breast cancer cells, including their mammosphere-forming abilities. UBQLN1 knockdown also enhanced breast cancer cell chemosensitivity to paclitaxel. The expression levels of the stem cell markers.
\end{abstract}

Correspondence to: Dr Xiaotang Yu or Professor Lianhong Li, Department of Pathology and Forensic Medicine, College of Basic Medical Science, Dalian Medical University, 9 Lvshun South Road, Dalian, Liaoning 116044, P.R. China

E-mail: xiaotang_yu@outlook.com

E-mail: lilianhong917@163.com

Abbreviations: UBQLN1, ubiquilin-1; CSCs, cancer stem cells; BCSCs, breast cancer stem cells; EMT, epithelial-to-mesenchymal transition; RT-qPCR, reverse transcription-quantitative PCR; MACS, magnetic-activated cell sorting; IGF1R, insulin like growth factor 1 receptor; PLIC, protein linking IAP with cytoskeleton; IAP, integrin-associated protein

Key words: ubiquilin-1, breast cancer, metastasis, epithelial-tomesenchymal transition, breast cancer stem cells, AKT signaling
Aldehyde dehydrogenase 1 (ALDH1), Oct-4 and Sox 2 were significantly decreased in the cells in which UBQLN1 was silenced, whereas breast cancer stem cells exhibited an increased expression of UBQLN1. Mechanistically, UBQLN1 knockdown inhibited the activation of AKT signaling, as revealed by the increased PTEN expression and the decreased expression of phosphorylated AKT in cells in which UBQLN1 was silenced. On the whole, the present study demonstrates that UBQLN1 is aberrantly upregulated in breast cancer and predicts a poor prognosis. The silencing of UBQLN1 inhibited the invasion, EMT and stemness of breast cancer cells, possibly via AKT signaling.

\section{Introduction}

Breast cancer is the most commonly diagnosed type of cancer and is a prevailing cause of cancer-associated mortality among women. As previously reported, female breast cancer, with an estimated 2.3 million new cases (11.7\%) and 684,996 cancer-associated mortalities globally,surpassed lung cancer as the most commonly diagnosed cancer type in 2020 (1). The majority of breast cancer-associated mortalities are caused by distant metastasis (2). Decades of research on cancer have led to a substantial progress in the treatment of primary breast tumors; however, treatment options for metastatic cancer remain limited (3).

Accumulating evidence has revealed that cancer stem cells (CSCs), which constitute a small number of tumor cells in liquid and solid tumors, possess self-renewal capability and contribute to tumor onset, resistance, recurrence and metastasis (4). Breast CSCs (BCSCs) can be identified by various functional assays, including tumor sphere formation, xenograft assay or detection of specific cell-surface markers such as CD44, CD24, Oct-4 and aldehyde dehydrogenase (ALDH) (5). Another important factor contributing to metastasis is epithelial-to-mesenchymal transition (EMT), a process during which epithelial cells lose their polarity and cell-to-cell contact, acquiring additional migratory and invasive properties (6), which allows cancer cells to detach from neighboring cells, dissolve the basal membrane and invade the extracellular matrix (ECM) (7). Several molecular mechanisms are 
known to promote EMT, including the regulation of specific cell-surface protein and ECM-degrading enzyme expression levels, and the alteration of the expression of certain transcription factors. The EMT process during breast carcinogenesis is considered to be controlled by a series of signaling pathways, including PI3K/AKT (7), Notch (8), Wnt/ $\beta$-catenin (9) and Hedgehog $(4,8)$, being also responsible for CSC maintenance $(4,10)$.

Ubiquilin-1 (UBQLN1) belongs to a family of ubiquitin-like proteins that contain five major UBQLN proteins (UBQLN1-4 and UBQLNL). These proteins are evolutionarily conserved and structurally similar, containing an amino-terminal ubiquitin-like (UBL) domain, a carboxy-terminal ubiquitin-associated (UBA) domain and a series of four chaperonin-like domains within the central part of the protein $(6,11)$. The UBL domain mediates interaction with the proteasome, whereas the UBA domain preferentially binds to ubiquitinated proteins (12). UBQLNs appear to function as adaptors to deliver ubiquitinated proteins to the proteasome (13). UBQLNs are essential factors for the maintenance of proteostasis in cells, since they are important for the regulation of different protein degradation mechanisms and pathways, including the ubiquitin-proteasome system (UPS), autophagy and endoplasmic reticulum-associated protein degradation pathways (6). The $U B Q L N 1$ gene is located on human chromosome $9 q 22$ and is ubiquitously expressed in all human tissues (14). The role of UBQLN1 has mainly been studied in neurological disorders. The disruption of UBQLN1 function has been reported in a variety of neurological disorders caused by aberrant protein aggregation, such as Alzheimer's disease (15), amyotrophic lateral sclerosis (16) and Huntington's disease (17).

Previous studies have indicated that UBQLN1 participates in the progression of certain types of cancer, including lung adenocarcinoma (18), gastric adenocarcinoma (12) and ovarian cancer (19). Elevated UBQLN1 levels have been revealed to be associated with a poor prognosis of patients with breast cancer (20), lung and gastric cancer (12). UBQLN1 has also been reported to be associated with the stemness of cells such as human neuronal stem cell line (21) and human embryonic stem cells (22). However, UBQLN1 expression, and biological function and mechanisms in breast cancer remain largely unknown. In the present study, UBQLN1 expression in breast cancer tissues was examined, its clinical significance was explored, the effect of UBQLN1 knockdown on cell migration, invasion, EMT and stemness was investigated and the possible mechanisms of UBQLN1 in the progression of breast cancer were evaluated.

\section{Materials and methods}

Cells and cell culture. The human normal mammary epithelial cell line, MCF-10A (SCSP-575), and the human breast cancer cell lines, MCF-7 (SCSP-531) and MDA-MB-231 (SCSP-5043), were obtained from The Cell Bank of Type Culture Collection of the Chinese Academy of Sciences. The MCF-7 cell line was cultured in DMEM/F12 (HyClone; Cytiva) with $10 \%$ FBS (Gibco; Thermo Fisher Scientific, Inc.). MDA-MB-231 cells were cultured in MEM $\alpha$ modification medium (HyClone; Cytiva) supplemented with 10\% FBS.
MCF-10A cells were maintained in DMEM/F12 supplemented with $10 \% \mathrm{FBS}, 20 \mathrm{ng} / \mathrm{ml}$ human recombinant epidermal growth factor (R\&D Systems, Inc.), $0.5 \mu \mathrm{g} / \mathrm{ml}$ hydrocortisone (Sigma-Aldrich; Merck KGaA), $10 \mu \mathrm{g} / \mathrm{ml}$ insulin (Sigma-Aldrich; Merck KGaA), $0.1 \mu \mathrm{g} / \mathrm{ml}$ cholera toxin (Sigma-Aldrich; Merck KGaA) and L-glutamine (Invitrogen; Thermo Fisher Scientific, Inc.). Cells were maintained at $37^{\circ} \mathrm{C}$ and $5 \% \mathrm{CO}_{2}$.

Lentiviral construction and virus infection. Lentiviral vectors, including short hairpin RNA (shRNA/sh) against UBQLN1 (shUbqln1) and negative control shRNA (shScramble) were constructed by Shanghai GenePharma Co., Ltd. (LV3-UQLNQ1-homo1642). The sequence of shUbqln1 was as follows: 5'-GAG TAC TAC TGC GCC AAA T-3'. The sequence of shScramble was as follows: Sence, 5'-UUC UCC GAA CGU GUC ACG UTT-3'; antisense, 5'-ACG UGA CAC GUU CGG AGA ATT-3'. To silence UBQLN1, the MCF-7 and MDA-MB-231 cells were cultured in serum-free medium containing lentivirus (multiplicity of infection, 50) and $5 \mu \mathrm{g} / \mathrm{ml}$ Polybrene (GenePharma Co., Ltd.) for $6 \mathrm{~h}$ and then incubated in complete growth medium for $48 \mathrm{~h}$ at $37^{\circ} \mathrm{C}$. The minimal lethal concentration of puromycin (Clontech Laboratories, Inc.) $(10 \mu \mathrm{g} / \mathrm{ml})$ was used to selected stably transfected cells. Knockdown efficiency was determined by the use of western blot analysis and reverse transcription-quantitative PCR (RT-qPCR).

Isolation of $\mathrm{CD} 44^{+} / \mathrm{CD} 24^{-} \mathrm{BCSC}$. CD $44^{+} / \mathrm{CD} 24^{-} \mathrm{BCSC}$ were separated using the magnetic-activated cell sorting system (MACS) (Miltenyi Biotec GmbH) with CD44 MicroBeads and CD24 Microbeads kit (both from Miltenyi Biotec $\mathrm{GmbH}$ ), according to the manufacturer's protocol. Breast cancer cells were collected and resuspended in $40 \mu \mathrm{l}$ PBS with $0.5 \%$ BSA (Beyotime Institute of Biotechnology) and 2 mM EDTA (PBE) per $10^{7}$ cells, incubated with CD24-biotin and then incubated with anti-biotin microbeads following the manufacturer's protocol of the CD24 Microbeads kit. Subsequently, the cells were magnetically separated. Briefly, the cell suspension was applied to MiniMACS columns (Miltenyi Biotec $\mathrm{GmbH}$ ) in the magnetic field of the MACS Separator (Miltenyi Biotec $\mathrm{GmbH})$. Unlabeled cells (CD24-) were obtained by collecting the flow-through cells, while labeled cells (CD24+ cells) were collected by pipetting $1 \mathrm{ml}$ of PBE buffer onto the column. CD24- cells were incubated with CD 44 MicroBeads at $4^{\circ} \mathrm{C}$ for 15 min. Subsequently, the cells were magnetically separated again. $\mathrm{CD} 44^{+} / \mathrm{CD} 24^{-}$cells were obtained by collecting labeled cells from the column.

MTT assay. For the detection of cell viability, transfected MCF-7 and MDA-MB-231 cells were seeded in 96-well plates, at a density of $2 \times 10^{3}$ cells/well. Cell viability was assessed by MTT assay following $0,24,48$ and $72 \mathrm{~h}$ of incubation at $37^{\circ} \mathrm{C}$. For the evaluation of cell viability, the transfected cancer cells were seeded in 96-well plates at a density of $2 \times 10^{4}$ cells/well. Cell viability in each group was assessed by MTT assay following the addition of various concentrations $(0,5,10,50$ and $100 \mathrm{ng} / \mathrm{ml})$ of paclitaxel (Beyotime Institute of Biotechnology) and incubation at $37^{\circ} \mathrm{C}$ for $48 \mathrm{~h}$. Subsequently, MTT (Beyotime Institute of Biotechnology) was added to each 
well at a final concentration of $5 \mathrm{mg} / \mathrm{ml}$ and the cells were incubated $4 \mathrm{~h}$ at $37^{\circ} \mathrm{C}$. The medium was then aspirated, followed by the addition of $100 \mu \mathrm{l}$ DMSO to each well. The plates were then agitated for $10 \mathrm{~min}$ at room temperature, and the absorbance at $490 \mathrm{~nm}$ was measured using a spectrophotometer (Thermal Fisher Scientific, Inc.). Each value represented the mean \pm SEM. Responses to drug treatment were assessed by normalizing the treatment groups to the untreated controls.

Protein extraction and western blot analysis. Cells $\left(1 \times 10^{6}\right)$ were washed with cold PBS and harvested. Total protein lysate was prepared by cell sonication in ice-cold standard RIPA lysis buffer (Sigma-Aldrich; Merck KGaA) with proteinase and phosphatase inhibitors (Santa Cruz Biotechnology, Inc.) for $10 \mathrm{sec}$. The protein concentration was measured using the Easy II Protein Quantitative kit (TransGen Biotech Co., Ltd.). Protein lysate (50-80 $\mu \mathrm{g}$ ) was separated by 8-12\% SDS-PAGE and transferred onto PVDF membranes (MilliporeSigma). After blocking in a 5\% skimmed milk solution in TBS containing $0.05 \%$ Tween-20 (TBST) buffer for $1 \mathrm{~h}$ at room temperature, the membranes were incubated with the corresponding specific antibody solution at $4^{\circ} \mathrm{C}$ overnight and then with IRDye ${ }^{\circledR} 800 \mathrm{CW}$ goat anti-rabbit second antibody (cat. no. 926-82211; LI-COR Biosciences; $1: 16,000$ ) at $37^{\circ} \mathrm{C}$ for $1 \mathrm{~h}$. The immunoreactive bands were detected and analyzed using an Odyssey infrared imaging system 3.0 (LI-COR Biosciences). Protein expression levels were normalized to GAPDH expression levels.

The specific antibodies used for western blot analysis were as follows: Rabbit polyclonal anti-human UBQLN1 (cat. no. ab3341; Abcam; 1:500); rabbit monoclonal anti-human phosphorylated (p)-AKT (Ser473) (cat. no. 4060; Cell Signaling Technology, Inc.; 1:500); monoclonal anti-human GAPDH (cat. no. sc-47724; Santa Cruz Biotechnology, Inc.; 1:10,000); and anti-PTEN (cat. no. 22034-1-AP; 1:500), anti-AKT (cat.no. 10176-2-AP; 1:1,000) anti-Oct-4 (cat. no. 11263; 1:500), anti-Sox 2 (cat. no. 11064; 1:500), anti-ALDH1 (cat. no. 15910; 1:500), anti-MMP2 (cat. no. 10373; 1:1,000), anti-MMP9 (cat. no. 27306; 1:1,000), anti-Snail (cat. no. 13099; 1:500), anti-Twist (cat. no. 25465; 1:500), anti-Bcl-2 (cat. no. 12789; 1:500), anti-Bax (cat. no. 60267-1-Ig; 1:500), anti-E-cadherin (cat. no. 20874; 1:1,000), anti-vimentin (cat. no. 10366-1-AP; 1:1,000), anti-caspase-3 (cat. no. 19677; 1:500), anti-caspase-9 (cat. no. 10380; 1:500) and anti-N-cadherin (cat. no. 22018; 1:500) (all rabbit polyclonal anti-human antibodies were purchased from ProteinTech Group, Inc.

RNA extraction and RT-qPCR analysis. Total RNA was extracted from $1 \times 10^{6}$ cells using TRIzol ${ }^{\circledR}$ reagent (TransGen Biotech Co., Ltd.). Total RNA (1 $\mu \mathrm{g})$ was reverse transcribed into cDNA using TransScript ${ }^{\circledR}$ All-in-One First-Strand cDNA Synthesis SuperMiX kit (TransGen Biotech Co., Ltd.). The resulting cDNA samples were amplified by qPCR with SYBR Premix Ex Taq Master MIx kit (TransGen Biotech Co., Ltd.) in a 20- $\mu 1$ reaction mixture using an $\mathrm{iCycler}_{\mathrm{iQ}}^{\mathrm{TM}}$ Real Time PCR Detection System (Agilent Technologies, Inc.). The PCR cycles were performed as follows: One cycle at $94^{\circ} \mathrm{C}$ for $10 \mathrm{~min}, 40$ cycles at $95^{\circ} \mathrm{C}$ for $5 \mathrm{sec}, 60^{\circ} \mathrm{C}$ for $15 \mathrm{sec}, 72^{\circ} \mathrm{C}$ for $10 \mathrm{sec}$, and one final cycle at $72^{\circ} \mathrm{C}$ for $10 \mathrm{~min}$, followed by cooling to $4^{\circ} \mathrm{C}$. The primers were synthesized by Invitrogen
(Thermo Fisher Scientific, Inc.), and the sequences were as follows: UBQLN1 forward, 5'-GAA CCA GGA CCG AGC TTG A-3' and reverse, 5'-TGT ATT GCT CAC CAA GGA AGC A-3'; ALDH1 forward, 5'-TGC AGG TTG GGC TGA CAA-3' and reverse, 5'-GCA GGC CCT ATC TTC CAA ATG-3'; and GAPDH forward, 5'-GCA CCG TCA AGG CTG AGA AC-3' and reverse, 5'-TGG TGA AGA CGC CAG TGG A-3'. The relative expression levels of mRNA were normalized to the internal control (GAPDH), and fold-changes were calculated using the $2^{-\Delta \Delta \mathrm{Cq}}$ method (23).

Tumor cell migration and invasion assays. Invasion assays were performed using 24-well Transwell chambers $(8.0-\mu \mathrm{m}$ pore size; Corning, Inc.). Each Transwell chamber was coated with Matrigel matrix (Corning, Inc.) (100 $\mu \mathrm{l}$ at a dilution of 1:3 in DMEM; BD Biosciences), $24 \mathrm{~h}$ prior to use. Cells were cultured in DMEM for $24 \mathrm{~h}$ and then seeded onto cell inserts ( $2 \times 10^{4}$ cells/insert) in the upper chamber. Serum-free DMEM was added to the upper chamber, while DMEM $(0.5 \mathrm{ml})$ containing $10 \%$ FBS was added to the lower chamber. After $24 \mathrm{~h}$, the upper cells were removed, and the cells on the surface of the bottom chamber were fixed with methanol for $15 \mathrm{~min}$ and stained with $0.01 \%$ crystal violet (MedChemExpress) for $30 \mathrm{~min}$ at room temperature. Randomly selected areas were imaged with an inverted microscope (Olympus IX73; Olympus Corporation) connected to an Olympus DP73 camera (Olympus Corporation), and the number of cells was counted. The results represent the mean number of cells in five fields per membrane for triplicate inserts. Cell migration assays were conducted as the invasion assays, with the exception of the Matrigel coating.

Wound-healing assay. When cultured cells reached 70-80\% confluence in 6 -well plates, a $100-\mu 1$ pipette tip was used to produce scratches in the cell monolayer. Subsequently, the cells were washed with PBS and cultured in DMEM without FBS for $\leq 72 \mathrm{~h}$. Images were observed and captured at 0,24 , 48 and $72 \mathrm{~h}$ with an inverted microscope (Olympus IX73; Olympus Corporation) connected to an Olympus DP73 camera (Olympus Corporation). All assays were performed in triplicate.

Colony formation assay. Breast cancer cells were plated at a density of 1,000 cells/well in a 6 -well plate. Following 2 weeks of incubation at $37^{\circ} \mathrm{C}$ in complete medium, colonies were fixed with methanol for $15 \mathrm{~min}$ and stained with $0.01 \%$ crystal violet for $30 \mathrm{~min}$ at room temperature. The clones were then counted and imaged using an inverted microscope (Olympus IX73; Olympus Corporation) connected to an Olympus DP73 camera (Olympus Corporation). The holoclones were counted as previously reported (5).

Immunofluorescence analysis. Cells were cultured on a cover slide in 24 -well plates at $37^{\circ} \mathrm{C}$, fixed in $4 \%$ paraformaldehyde for $15 \mathrm{~min}$ and permeabilized with $100 \%$ ice-cold methanol at $-20^{\circ} \mathrm{C}$ for $10 \mathrm{~min}$. After washing with PBS, the cells were blocked in 5\% BSA (Beyotime Institute of Biotechnology) containing $0.3 \%$ Triton $^{\mathrm{TM}} \mathrm{X}-100$ for $2 \mathrm{~h}$ at $4^{\circ} \mathrm{C}$. The cells were then washed with TBST and incubated overnight at $4^{\circ} \mathrm{C}$ with primary antibodies against UBQLN1 (cat. no. ab3341; Abcam; 1:100), p-AKT (cat. no. 4060; Cell Signaling Technology, 
Inc.; 1:100), PTEN (cat. no. 22034; 1:100), E-cadherin (cat.no. 20874; ProteinTech Group, Inc.; 1:100) and N-cadherin (cat. no. 22018; ProteinTech Group, Inc.; 1:100). Subsequently, the cells were incubated with a FITC-conjugated secondary antibody (cat. no. 408308; BioLegend, Inc.) for $1 \mathrm{~h}$ at $4^{\circ} \mathrm{C}$. Cell nuclei were counterstained with DAPI (Boster Biological Technology). Cells were then observed under an Olympus BX41 fluorescence microscope (Olympus Corporation) and photographed using an Olympus DP72 camera (Olympus Corporation).

Mammosphere formation assay. MCF-7 and MDA-MB-231 cells were inoculated into ultralow attachment 6-well plates (Corning, Inc.) at a density of $4 \times 10^{4}$ cells/well. The cells were grown in complete medium supplemented with B27 (1:50; Invitrogen; Thermo Fisher Scientific, Inc.), $5 \mu \mathrm{g} / \mathrm{ml}$ insulin (Sigma-Aldrich; Merck KGaA), $20 \mathrm{ng} / \mathrm{ml}$ human EGF (Sigma-Aldrich; Merck KGaA), $4 \mu \mathrm{g} / \mathrm{ml}$ heparin (Sigma-Aldrich; Merck KGaA) and $20 \mathrm{ng} / \mathrm{ml}$ basic fibroblast growth factor (Sigma-Aldrich; Merck KGaA) for 14 days. Cell colonies $>60 \mu \mathrm{m}$ in diameter were counted under an inverted microscope (Olympus Corporation).

Immunohistochemistry (IHC). A tissue array slide containing 73 cases of breast cancer tissues with complete clinicopathological data including age, tumor diameter, lymph node metastasis, TNM stage, histology, differentiation, molecular subtypes, estrogen receptor, progesterone receptor, HER2 and proliferating cell nuclear antigen expression, was used as reported previously (24). The mean age of the patients was $52.22 \pm 10.79$ years with a range between 29 and 81 years. A total of 30 normal breast tissues were collected from patients with fibrocystic breast disease. The breast cancer tissues and breast tissues were obtained by tumor resection surgery at the Affiliated Hospital of Dalian Medical University. Written informed consent was obtained from all individual participants included in the study. The present study was conducted according to the principles of the Declaration of Helsinki and was reviewed and approved by the Ethics Committee and Institutional Review Board (IRB) of Dalian Medical University (IRB approval no. 2021006).

Tissue sections were routinely deparaffinized in xylene and rehydrated in a series of $100-50 \%$ ethanol solutions. Endogenous peroxidase activity was blocked by incubation with $3 \%$ hydrogen peroxide in PBS for $15 \mathrm{~min}$ at room temperature. The tissue sections were then blocked with $10 \%$ normal goat serum (OriGene Technologies, Inc.) for $1 \mathrm{~h}$ at room temperature and then incubated with a primary antibody against UBQLN1 (cat. no. ab3341; Abcam) (1:100) overnight at $4^{\circ} \mathrm{C}$. A biotin-streptavidin HRP and AP detection for mouse and rabbit antibody on human tissue (cat. no. D03-6; OriGene Technologies, Inc.) was used for immunodetection following the manufacturer's instructions. In brief, all slides were incubated with a secondary antibody for $1 \mathrm{~h}$ at $4{ }^{\circ} \mathrm{C}$ and then with a streptavidin-biotin solution for $30 \mathrm{~min}$ at room temperature. The sections were then subjected to a colorimetric reaction using a DAB detection kit (OriGene Technologies, Inc.). Finally, the sections were briefly counterstained with hematoxylin. A negative control staining was also performed, by replacing the primary antibodies with normal goat serum
(OriGene Technologies, Inc.). The results of IHC were blindly evaluated by two independent pathologists. The scoring criteria were based on staining intensity (1, no or weak staining; or 2, medium or strong staining). The immunostained tissues were scored by multiplying the intensity (1-2) and extent (0-100\%) of staining (25). Total score cut-offs of $\leq 100$ and $>100$ were used to divide patients into low and high UBQLN1 expression groups, respectively.

Bioinformatics analysis. TNMplot (https://www.tnmplot. com/), Kaplan-Meier plotter (KmPlot) (http://kmplot. com/analysis/), cBioPortal (http://www.cbioportal.org), TIMER2.0 (http://timer.cistrome.org) and UALCAN (http://ualcan.path.uab.edu) were utilized to analyze the differential expression, prognostic value, genetic alteration, gene promotor methylation and clinicopathological significance of UBQLN1 in patients with breast cancer. TIMER2.0 was used to evaluate the outcome significance of UBQLN1 mRNA expression, optionally adjusted by clinical factors, including age, sex, stage and race using the Cox proportional hazard model. A threshold of $\mathrm{P}<0.05$ was used to set the cut-off criterion. A protein-protein interaction (PPI) network was obtained using STRING database (http://string-db.org).

Statistical analysis. Statistical analysis was performed using SPSS version 19.0 (IBM Corp.) and GraphPad Prism 5 (GraphPad Software, Inc.). Associations between clinicopathological characteristics and UBQLN1 expression were examined using the $\chi^{2}$ test. Differences between two groups were evaluated using a paired or unpaired Student's t-test. One-way ANOVA and Dunnett's multiple comparisons test were used for comparing multiple groups. Each set of results represents $\geq 3$ separate experiments. All experimental data are expressed as the mean $\pm \mathrm{SE}$. $\mathrm{P}<0.05$ was considered to indicate a statistically significant difference.

\section{Results}

$U B Q L N 1$ expression is significantly increased in breast cancer, and a high UBQLN1 expression is associated with a poor prognosis of patients with breast cancer. UBQLN1 is abnormally expressed in certain cancer types, including lung adenocarcinoma $(11,18)$ and gastric cancer $(12)$. In the present study, to elucidate UBQLN1 expression in breast cancer, UBQLN1 expression was detected in a tissue array slide, containing samples from 73 breast cancer cases and 30 normal breast tissues by using IHC. UBQLN1 immunoreactivity was mainly observed in the cytoplasm. expression in breast cancer tissues was significantly higher than normal breast tissue UBQLN1 expression (Fig. 1A-C).

The association between UBQLN1 expression and clinicopathological features of patients with breast cancer was further investigated. The results demonstrated that UBQLN1 expression was significantly associated with tumor diameter $(\mathrm{P}=0.034)$, lymph node metastasis $(\mathrm{P}=0.016)$ and $\mathrm{TNM}$ staging $(\mathrm{P}=0.047)$ (Fig. 1D and Table I); however, UBQLN1 expression was not significantly associated with age, histology, differentiation, molecular subtypes, or estrogen receptor, progesterone receptor, HER2 or proliferating cell nuclear antigen expression (Table I). Breast cancer cases with a higher 
A

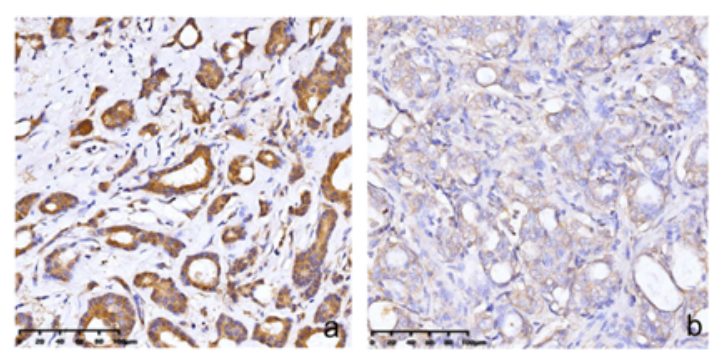

D

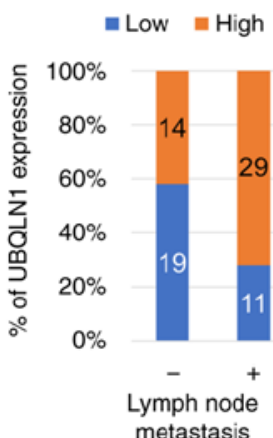

G

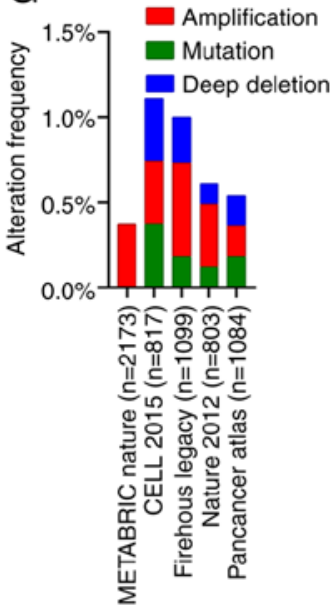

B

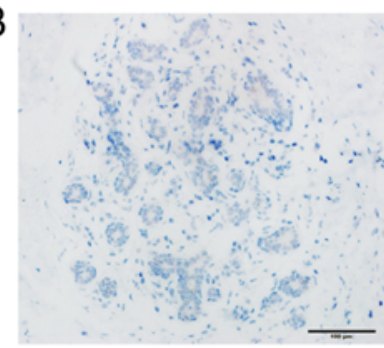

E

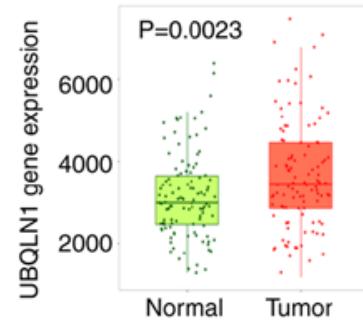

C

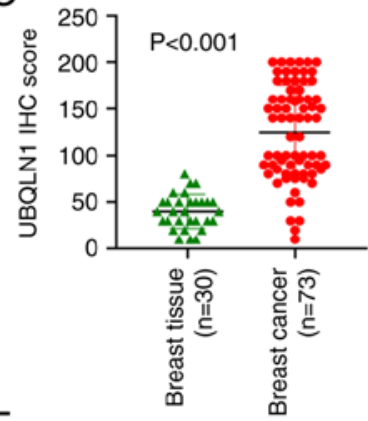

F

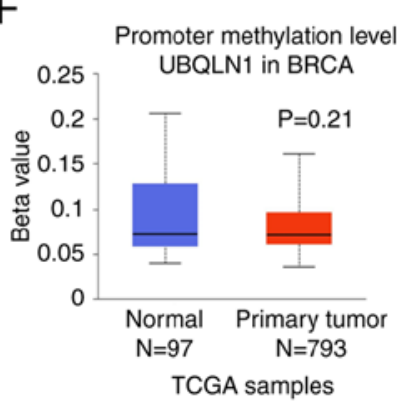

I

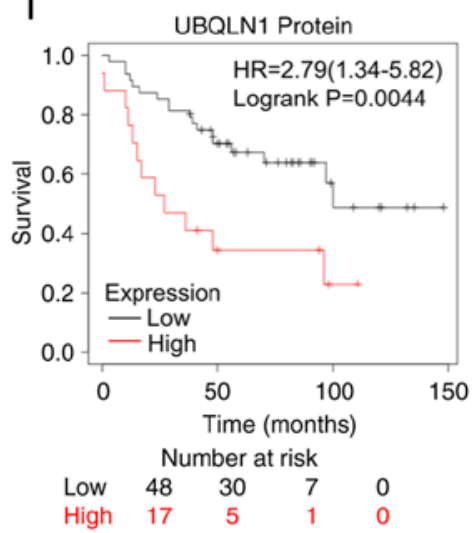

Median survival (Tang 2018)

\begin{tabular}{|c|c|}
\hline $\begin{array}{c}\text { Low expression } \\
\text { cohort (months) }\end{array}$ & $\begin{array}{c}\text { High expression } \\
\text { cohort (months) }\end{array}$ \\
\hline 100 & 27 \\
\hline
\end{tabular}

Figure 1. UBQLN1 is highly expressed in breast cancer tissues and is associated with a poor prognosis of patients with breast cancer. (A) Breast cancer with (a) strong or (b) weak UBQLN1 expression. (B) Weak expression of UBQLN1 in normal breast tissue. (C) UBQLN1 immunohistochemical staining scores in breast cancer tissues $(n=73)$ and breast tissues $(n=30)$. (D) Bar graphs showing the percentage of breast cancer with high and a low UBQLN1 expression in patients with and without lymph node metastasis, and with different TNM stages. The numbers in the bars indicate the case numbers. (E) UBQLN1 transcripts were evidently overexpressed in breast cancer tissues $(\mathrm{n}=112)$ compared with their expression levels in paired normal breast tissues from the TNMplot database. (F) UBQLN1 promoter methylation in normal breast tissues and breast cancer tissues (UALCAN). (G) Graphs indicating the frequency of UBQLN1 somatic mutations in breast cancer, as extracted from cancer studies in cBioPortal (mRNA expression z scores relative to diploid samples in tumors). (H and I) Kaplan-Meier diagrams showing the overall survival of patients with breast cancer depending on the expression of UBQLN1 (H) mRNA and (I) protein in kmplot.com (http://kmplot.com/analysis/). The patients were divided into the high and low expression groups according to the 'auto select best cutoff' provided by the Kaplan-Meier plotter server. UBQLN1, ubiquilin-1.

UBQLN1 expression tended to have a more advanced stage and to undergo lymphatic metastasis more frequently.

To further explore the clinical significance of UBQLN1 in breast cancer, a number of widely used databases were employed, in order to clarify whether this difference was associated with $U B Q L N 1$ promotor methylation. Using TNMPlot (26), it was revealed that UBQLN1 mRNA expression was significantly higher in breast cancer tissues than in paired normal breast tissues (Fig. 1E). Data from UALCAN indicated that there was no significant difference in UBQLN1 methylation between normal breast tissues and breast cancer tissues (Fig. 1F). The genomic alteration of $U B Q L N 1$ was further investigated using cBioPortal. The genetic alterations affecting UBQLN1 identified by cBioPortal in five breast cancer studies are shown in Fig. 1G. Gene mutations and gene number alterations were found in $39(<0.1 \%)$ out of 5,976 patients with breast cancer and the most frequent alteration was gene amplification (22 cases) (Table SI). Subsequently, mRNA expression in these breast cancer cases was investigated in relation to mean mRNA expression in diploid samples in breast cancer 
Table I. Association between UBQLN1 expression and clinicopathological parameters of breast ductal carcinoma cases.

\begin{tabular}{|c|c|c|c|c|c|}
\hline & & & UBQLN1 & sion, n (\%) & \\
\hline Chara & & No. of cases & Low $(n=30)$ & $\operatorname{High}(n=43)$ & P-value \\
\hline Age & $<50$ & 32 & $14(44)$ & $18(56)$ & 0.811 \\
\hline & $\geq 50$ & 41 & $16(39)$ & $25(61)$ & \\
\hline Diameter $(\mathrm{cm})$ & $<2$ & 31 & $18(58)$ & $15(48)$ & $0.034^{\mathrm{a}}$ \\
\hline & $\geq 2$ & 42 & $12(29)$ & $38(90)$ & \\
\hline LN & - & 33 & $19(58)$ & $14(42)$ & $0.016^{\mathrm{a}}$ \\
\hline & + & 40 & $11(28)$ & $29(73)$ & \\
\hline $\mathrm{DM}$ & - & 72 & $30(42)$ & $42(58)$ & 0.431 \\
\hline & + & 1 & $0(0)$ & $1(100)$ & \\
\hline TNM stage & I & 18 & $12(67)$ & $6(33)$ & $0.047^{\mathrm{a}}$ \\
\hline & II & 38 & $14(42)$ & $24(58)$ & \\
\hline & III & 16 & $4(25)$ & $12(75)$ & \\
\hline & IV & 1 & $0(0)$ & $1(100)$ & \\
\hline Grade & 1 & 12 & $6(50)$ & $6(50)$ & 0.388 \\
\hline & 2 & 48 & $17(35)$ & $31(65)$ & \\
\hline & 3 & 13 & $7(54)$ & $6(46)$ & \\
\hline ER & - & 40 & $19(48)$ & $21(53)$ & 0.242 \\
\hline & + & 33 & $11(33)$ & $22(67)$ & \\
\hline PR & - & 53 & $22(42)$ & $21(40)$ & 0.061 \\
\hline & + & 20 & $8(40)$ & $12(60)$ & \\
\hline Her-2 & - & 61 & $24(39)$ & $37(61)$ & 0.534 \\
\hline & + & 12 & $6(50)$ & $6(50)$ & \\
\hline Molecular subtype & Luminar A & 31 & $11(35)$ & $20(65)$ & 0.183 \\
\hline & Luminal B & 27 & $12(44)$ & $15(56)$ & \\
\hline & HER2-enriched & 7 & $4(57)$ & $3(43)$ & \\
\hline & Triple-negative & 8 & $3(37)$ & $5(63)$ & \\
\hline PCNA & - & 28 & $12(43)$ & $16(57)$ & 0.812 \\
\hline & + & 45 & $18(40)$ & $27(60)$ & \\
\hline
\end{tabular}

${ }^{\mathrm{a}} \mathrm{P}<0.05$. LN, lymph node metastasis; DM, distant metastasis; ER, estrogen receptor; PR, progesterone receptor; PCNA, proliferating cell nuclear antigen; UBQLN1, ubiquilin-1.

cases as follows: $223(5.00 \%)$ out of 4,462 cases demonstrated a higher expression, while $112(2.51 \%)$ cases exhibited a lower expression (Table SII). UALCAN revealed that UBQLN1 mRNA expression was significantly associated with tumor stage and lymph node metastasis (Table SIII).

Survival analysis using KmPlot revealed that, in breast cancer, patients with a low UBQLN1 mRNA expression had a longer median survival time (54 months) than patients with a high UBQLN1 expression (25 months) (Fig. 1H) (27). The analysis of data $(\mathrm{n}=118)$ from Tang et al $(28)$ revealed that UBQLN1 protein expression was also associated with a poor prognosis (Fig. 1I). TIMER2 was used with a Cox proportional hazard model to evaluate the significance of UBQLN1 mRNA expression adjusted by clinical factors, including age, sex, ethnicity and tumor stage in 976 patients with 136 dying. The results demonstrated that UBQLN1 [hazard ratio $(\mathrm{HR})=1.33$, $\mathrm{P}=0.038]$, age $(\mathrm{HR}=1.03, \mathrm{P}<0.001)$, stage $3(\mathrm{HR}=3.28, \mathrm{P}<0.001)$ and stage $4(\mathrm{HR}=14.17, \mathrm{P}<0.001)$ were significant predictors of survival (Table SIV). These data indicated a close association between UBQLN1 and lymph node metastasis. and suggested that UBQLN1 may be an independent prognostic predictor for overall survival of patients with breast cancer.

UBQLN1 knockdown attenuates breast cancer cell migration and invasion by inhibiting EMT. The results of the analysis of UBQLN1 in breast cancer tissues suggested that it may facilitate the metastasis of breast cancer. In the present study, the biological function of UBQLN1 in breast cancer cell lines was then investigated using in vitro experiments. UBQLN1 expression in breast cancer cells was first investigated using western blot analysis. Compared with that in the human normal breast epithelial cell line, MCF-10A, UBQLN1 expression was significantly higher in the breast cancer cell lines, MCF-7 and MDA-MB-231 (Fig. 2A).

Subsequently, the effect of UBQLN1 silencing on the biological behavior of breast cancer cell lines was investigated. The expression of UBQLN1 in the MCF-7 and MDA-MB-231 cell lines was stably knocked down using a lentivirus carrying UBQLN1 shRNA. UBQLN1 protein and mRNA expression was significantly inhibited in the MCF-7-shUbqln1 
A

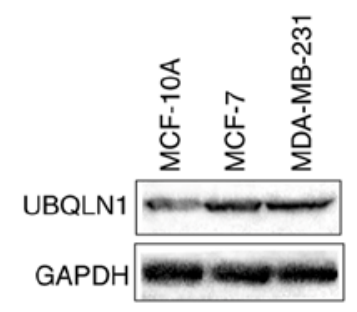

B

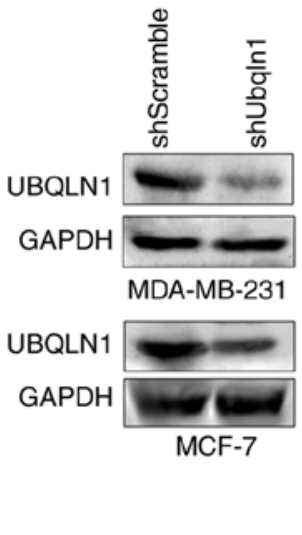

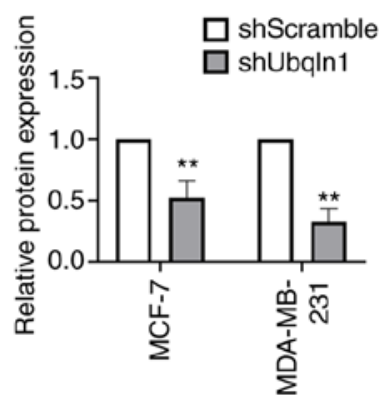

C
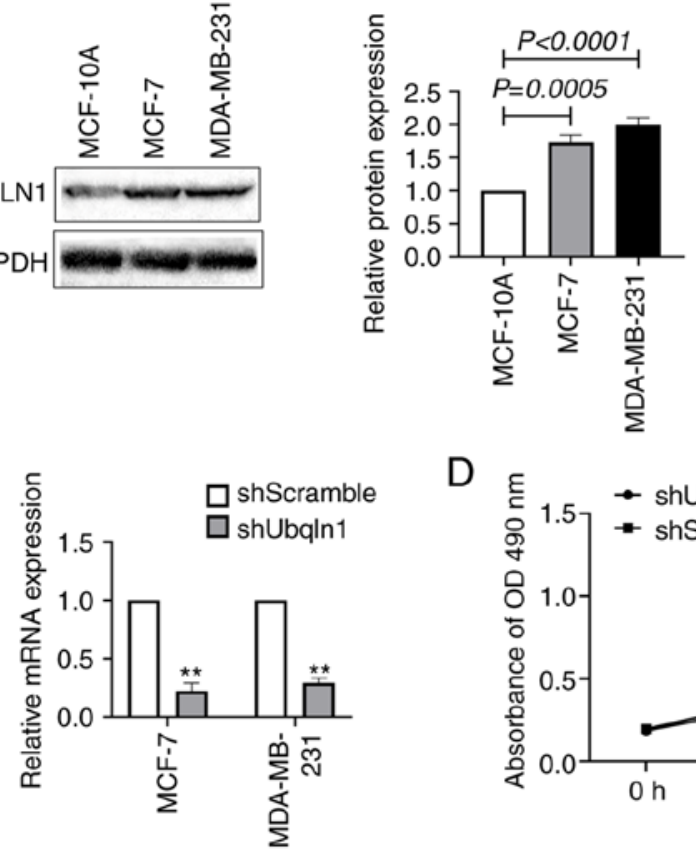

$\mathrm{D}$
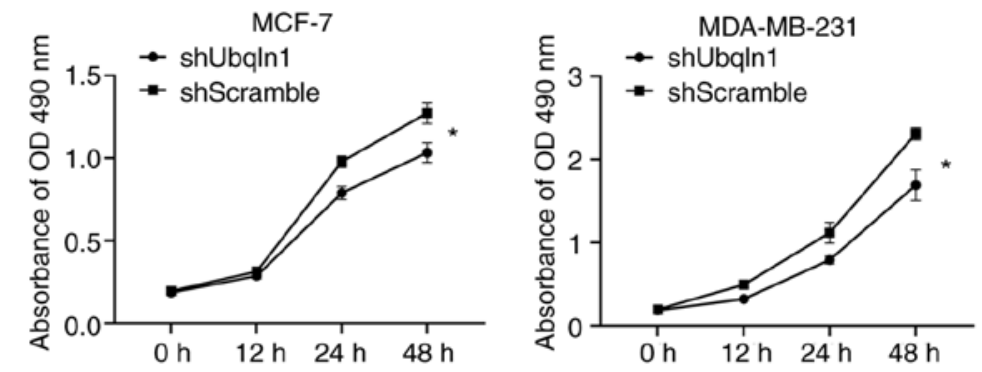

E

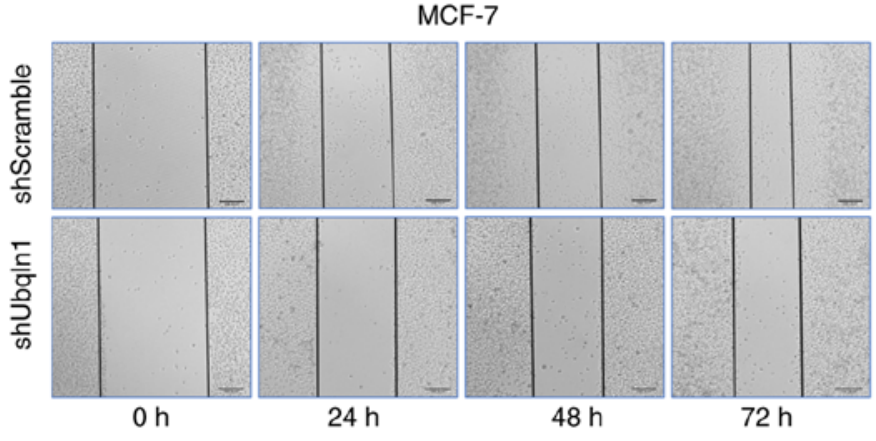

F
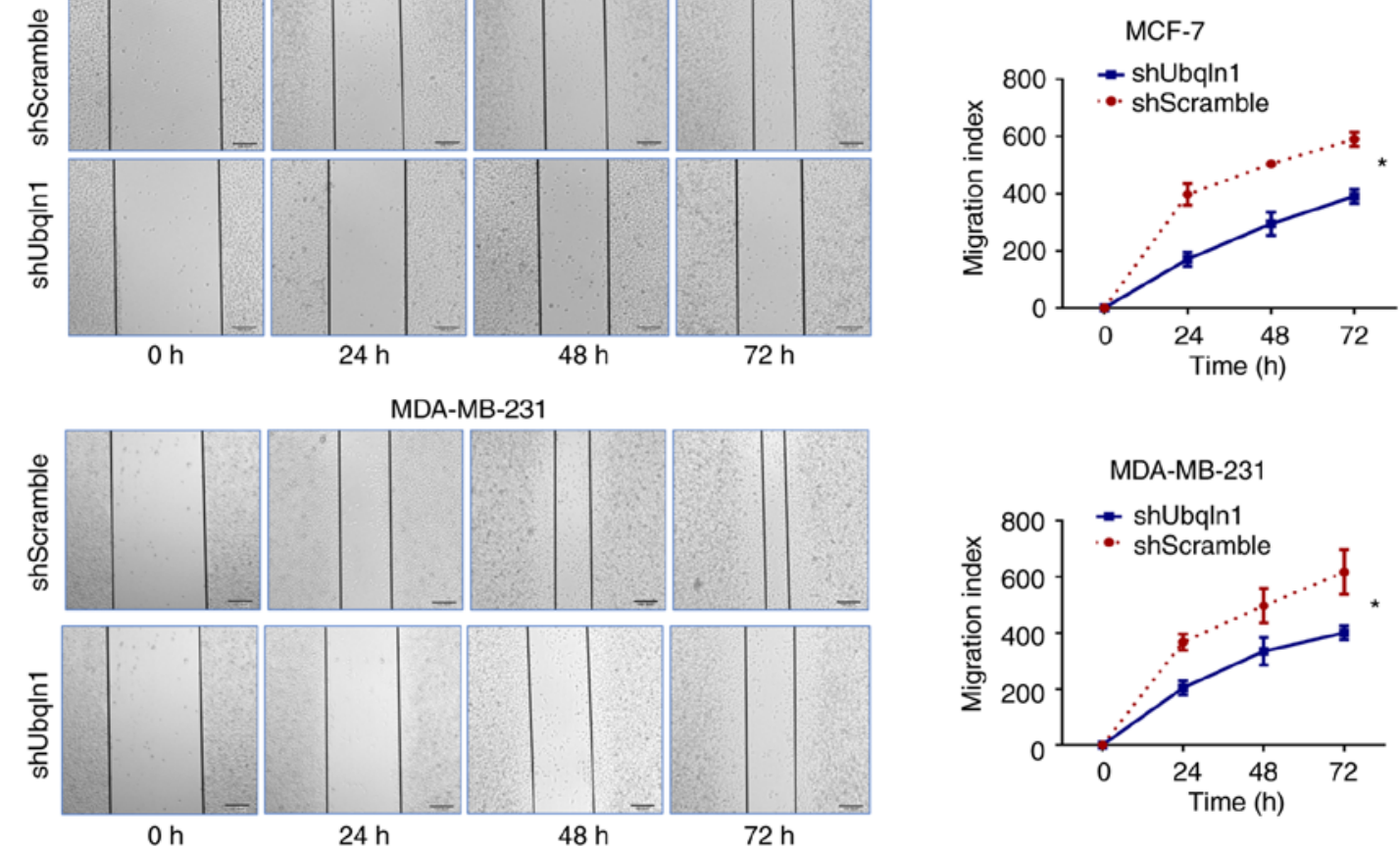

Figure 2. UBQLN1 knockdown inhibits the viability and migration of breast cancer cells. MDA-MB-231 and MCF-7 cells were stably transfected with lentivirus carrying UBQLN1 shRNA (shUbqln1) or control shRNA (shScramble) and then subjected to different assays. (A) Western blot analysis of UBQLN1 expression in normal breast epithelial cells (MCF-10A) and human breast cancer cells (MCF-7 and MDA-MB-231). Bar graphs show protein relative expression levels. (B) Western blot and (C) reverse transcription-quantitative PCR analyses of UBQLN1 knockdown efficiency in MCF-7 and MDA-MB-231 cells. (D) MTT cell viability assay of cells after incubation for 0,24,48 and $72 \mathrm{~h}$. (E) Representative microphotographs of the wound-healing assay. (F) Quantification of wound-healing assay data. Values represent the mean \pm SEM. ${ }^{*} \mathrm{P}<0.05$ and ${ }^{* *} \mathrm{P}<0.01$. UBQLN1, ubiquilin-1; shRNA, short hairpin RNA.

and MDA-MB-231-shUbqln1 cells compared with the expression levels in the controls (MCF-7-shScramble and MDA-MB-231-shScramble), as determined by western blot analysis (Fig. 2B) and RT-qPCR (Fig. 2C).

MTT assay recealed that MCF-7 and MDA-MB-231 cell viability was significantly inhibited when UBQLN1 was knocked down $(\mathrm{P}<0.05)$ (Fig. 2D). The effect of UBQLN1 knockdown on cell migration was investigated using wound healing assay and Transwell migration assays. The results indicated that UBQLN1 knockdown significantly suppressed cell migration (Figs. 2E and $\mathrm{F}$ and $3 \mathrm{~A}$ and $\mathrm{B}$ ). The effect of UBQLN1 knockdown on cell invasion was then investigated using Transwell invasion assay, which uses Matrigel to simulate the extracellular matrix. The results revealed that UBQLN1 
A
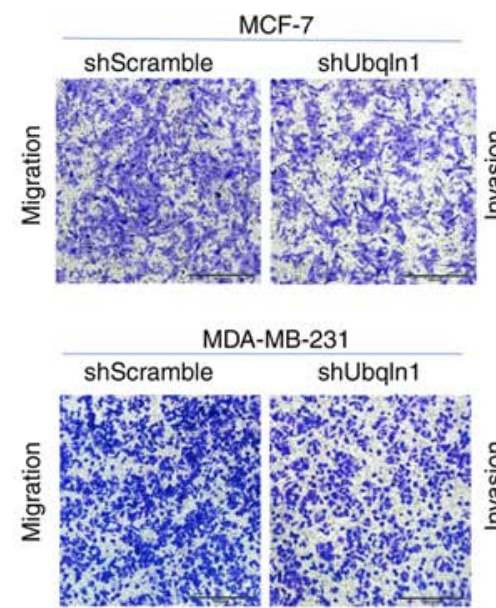

C
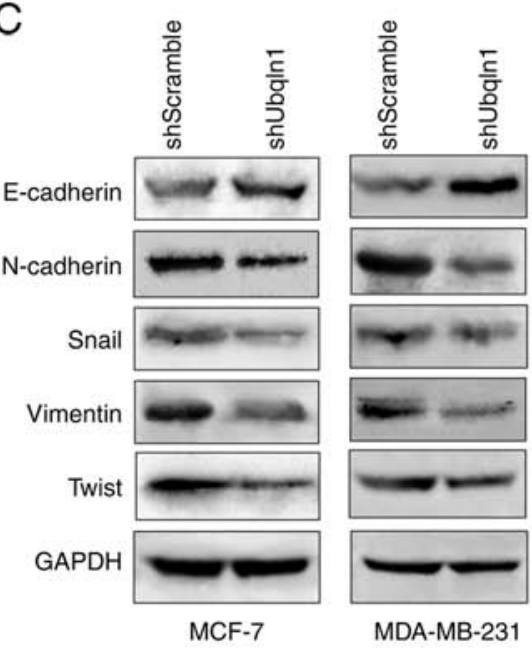
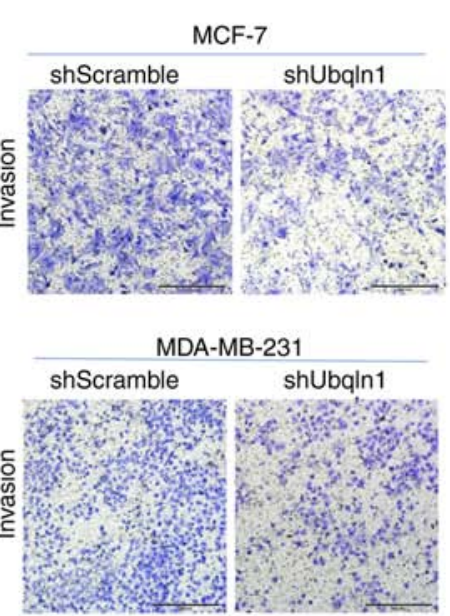

$\mathrm{B}$
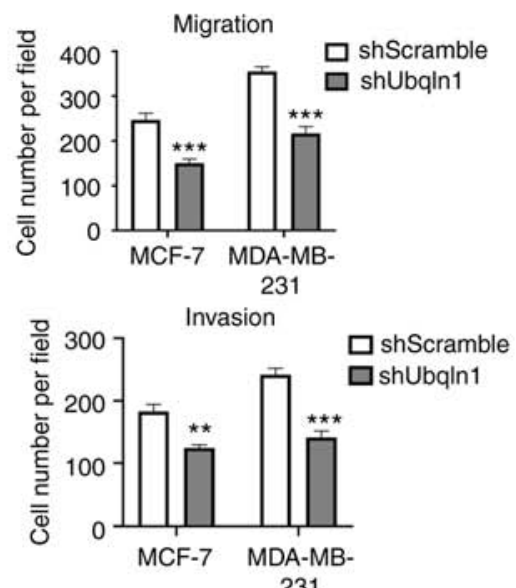

231

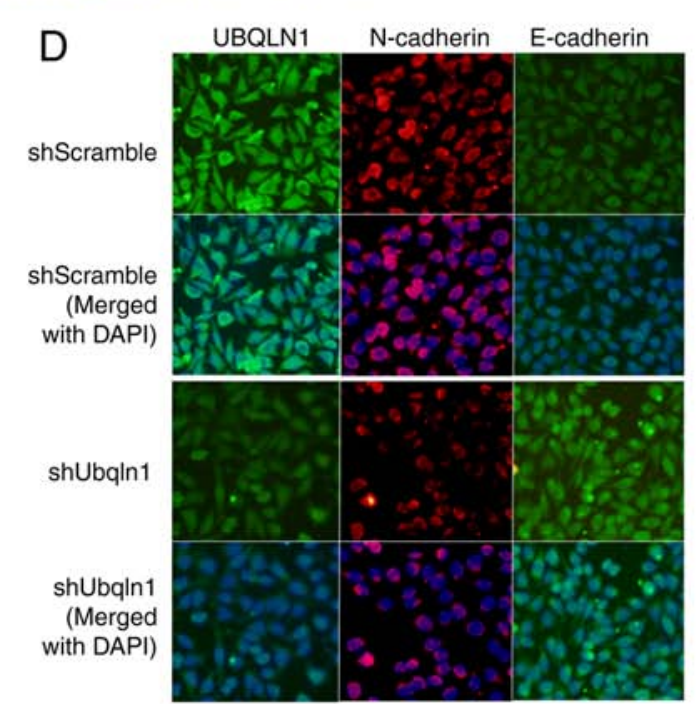

E
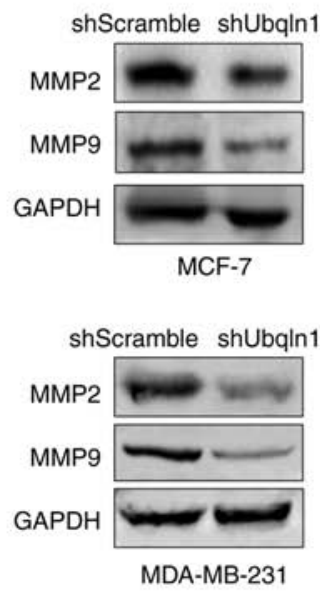

Figure 3. UBQLN1 knockdown inhibits the migration, invasion and epithelial-to-mesenchymal transition of breast cancer cells. (A) Representative microphotographs of tumor cell migration and invasion assays (Transwell assays). (B) Quantification of cell migration and invasion assays data. (C) Western blot analysis of vimentin, E-cadherin, N-cadherin, Snail and vimentin expression. (D) Representative microphotographs of immunofluorescence staining of E-cadherin and N-cadherin. (E) Western blot analysis of MMP2 and MMP9 expression. Values represent the mean \pm SEM. ${ }^{* *} \mathrm{P}<0.01$ and ${ }^{* * *} \mathrm{P}<0.001$. UBQLN1, ubiquilin-1; shRNA, short hairpin RNA.

knockdown significantly inhibited breast cancer cell invasion, as indicated by the number of invaded MCF-7-shUbqln1 and MDA-MB-231-shUbqln1 cells, which was decreased in comparison with that in MCF-7-shScramble $(\mathrm{P}<0.01)$ and MDA-MB-231-shScramble groups ( $\mathrm{P}<0.001)$ (Fig. 3A and $\mathrm{B})$.

Cancer cells acquire migratory and invasive abilities by EMT, through which, epithelial cells acquire enhanced mobility and invasive properties by losing cell-cell adhesion structures and polarity (29). The present study further investigated the effect of UBQLN1 knockdown on the expression of EMT markers. In the cells in which UBQLN1 was silenced, the expression of the epithelial marker, E-cadherin, was increased, while the expression of the mesenchymal markers, vimentin and $\mathrm{N}$-cadherin, was decreased. The expression of Snail, which is an E-cadherin repressor and a major EMT inducer, was markedly downregulated in the cells in which UBQLN1 was silenced (Fig. 3C). Similar results were observed using immunofluorescence analysis for $\mathrm{N}$-cadherin and E-cadherin expression (Fig. 3D). These findings indicated that UBQLN1 knockdown promoted mesenchymal-epithelial transition (MET) and effectively inhibited EMT in breast cancer.
MMP2 and MMP9, two important enzymes that degrade the extracellular matrix, are associated with EMT and the metastatic potential of cancer cells (30). In the present study, the effect of UBQLN1 knockdown on the expression of MMP2 and MMP9 was then investigated. Western blot analysis revealed that the expression of MMP2 and MMP9 was markedly decreased in the cells in which UBQLN1 was silenced, suggesting that UBQLN1 may promote invasion via the upregulation of MMP expression (Fig. 3E).

UBQLN1 promotes the stemness and chemoresistance of breast cancer cells. It has been reported that BCSCs possess self-renewal capabilities and contribute to tumor onset, recurrence, metastasis and therapy resistance (31). The present study investigated whether UBQLN1 is associated with the stem cell properties of breast cancer. Firstly, it was investigated whether then downregulation of UBQLN1 affects the expression of BCSC markers. The results revealed that ALDH1, Oct-4 and Sox 2 expression levels were markedly decreased in the breast cancer cells in which UBQLN1 was silenced $(\mathrm{P}<0.001$; Fig. 4A). 
$\mathrm{CD} 24^{-} / \mathrm{CD} 44^{+}$cells and $\mathrm{ALDH} 1^{+}$cells are widely considered to be BCSCs (24). Thus, in the present study, the expression of UBQLN1 in BCSCs was determined. The CD24 $/ \mathrm{CD} 44^{+}$cell population from the MDA-MB-231 and MCF-7 cell lines was isolated using MACS and confirmed by their higher expression of the BCSC marker ALDH1. The results of RT-qPCR demonstrated that UBQLN1 mRNA expression was significantly higher in the $\mathrm{CD} 24{ }^{-} / \mathrm{CD} 44^{+}$cells than in the CD24+ or CD24/CD44- cells (Fig. 4B).

Mammosphere formation reflects the self-renewal potential of tumor cells (31). In the present study, the results of mammosphere formation assays indicated that UBQLN1 knockdown significantly decreased the number of mammospheres $(\mathrm{P}<0.01$; Fig. 4C and D). Holoclone formation is a typical property of CSCs. The number of holoclones in MCF-7-shUbqln1 and MDA-MB-231-shUbqln1 cells was significantly lower than that in the control groups $(\mathrm{P}<0.01$; Fig. $4 \mathrm{E}$ and $\mathrm{F})$.

The effects of UBQLN1 knockdown on the sensitivity of breast cancer cells to chemotherapy were then investigated in the present study. Since paclitaxel is frequently used as the first-line treatment drug in breast cancer (32), the cells were treated with various concentrations of paclitaxel. MTT assay indicated that the MCF7-shUbqln1 and MDA-MB-231-shUbqln1 cells were more sensitive to paclitaxel than the controls $(\mathrm{P}<0.001$; Fig. 4G). The drug concentrations that inhibited cell proliferation by $50 \%\left(\mathrm{IC}_{50}\right)$ in the cells in which UBQLN1 was knocked down were significantly lower than those in the control cells $(\mathrm{P}<0.001$; Fig. $4 \mathrm{H})$. This result suggested that UBQLN1 knockdown promoted the cytotoxic effects of paclitaxel.

The results of western blot analysis demonstrated that UBQLN1 knockdown promoted paclitaxel-induced apoptosis. This was evidenced by the finding that cleaved caspase- 3 and caspase-9 expression was significantly upregulated in the MDA-MB-231 cells in which UBQLN1 was knocked down, and caspase-9 expression was upregulated in the MCF-7 cells in which UBQLN was knocked down and treated with paclitaxel for $48 \mathrm{~h}(\mathrm{P}<0.01$; Fig. 4I). Caspase-3 expression was not detected in MCF-7 cells as these cells do not express caspase-3 (33).

The expression of $\mathrm{Bcl}-2$, an anti-apoptotic member of the Bcl-2 protein family, was significantly decreased, while that of Bax, a pro-apoptotic effector protein, was increased in the cells in which UBQLN1 was knocked down (Fig. 4I). As a result, the $\mathrm{Bax} / \mathrm{Bcl}-2$ ratio significantly increased $(\mathrm{P}<0.05$; Fig. 4J), thus indicating that UBQLN1 effectively inhibited paclitaxel-induced apoptosis through Bcl-2 family members.

UBQLN1 sustains stemness and EMT by regulating PI3K/AKT signaling. UBQLN1 is important for the regulation of protein degradation. Beverly et al (34) determined that UBQLN1 was associated with specific biological functions or canonical biological pathways by Ingenuity Pathway Analysis (IPA), and found that AKT signaling is the most markedly canonical pathway represented by this gene. The present study investigated the effect of UBQLN1 on the expression of molecules involved in AKT signaling. AKT expression was not altered, while p-AKT expression was significantly decreased in the cells in which UBQLN1 was knocked down. PTEN, an inhibitor of AKT signaling, was significantly increased in
UBQLN1-silenced cells $(\mathrm{P}<0.05$; Fig. 5A and B). Similar results were also observed by immunofluorescence analysis. Following the loss of UBQLN1 in breast cancer, PTEN expression was increased, while p-AKT expression was decreased in the MDA-MB-231 cells (Fig. 5C).

Furthermore, PPI network analysis was conducted using STRING to analyze UBQLN1-related molecules, including EGFR, insulin like growth factor 1 receptor (IGF1R), mTOR, AKT, PTEN; stem cell markers, such as ALDH1, Oct-4 and Sox2; and EMT-related molecules, such as Twist, Snail, MMP2 and MMP9, and to explore the potential interactions among them. It was observed that these molecules were all closely associated with AKT signaling (Fig. 5D). Therefore, UBQLN1 may inhibit migration, invasion, EMT and the stemness of breast cancer by affecting the activation of PI3K/AKT signaling (Fig. 5E).

\section{Discussion}

In the present study, UBQLN1 expression and function in breast cancer tissues and cell lines was investigated. The findings for breast cancer tissues using IHC, and the data from UALCAN, KmPlot and TIMER2.0 demonstrated that UBQLN1 was highly expressed in breast cancer tissues and breast cancer cell lines both at the mRNA and protein level. UBQLN1 expression in breast cancer was associated with TNM stage, lymph node metastasis and a poor prognosis. These results suggested that UBQLN1 is associated with metastasis and may be an independent prognostic predictor for overall survival of patients with breast cancer. While the total number of cases included in the IHC analysis in present study was relatively small, including only 1 case with stage IV, further more well-characterized largescale studies are required to validate the association of UBQLN protein expression with the distant metastasis of breast cancer. UBQLN1 expression has been estimated in several cancer types, including non-small cell lung cancer (NSCLC) $(6,11,34-36)$, and breast (20), gastric (12) and ovarian cancer (19). The majority of the findings in the literature cited above are consistent with the findings of the present study, suggesting that UBQLN1 promotes cancer progression and may play an oncogenic role in cancer. In gastric and breast cancer, UBQLN1 has been found to be highly expressed in cancer tissues, and to be positively associated with TNM stage, tumor invasion and lymph node metastasis. Higher UBQLN1 expression levels have been shown to be associated with a shorter survival of patients with gastric cancer and breast cancer $(12,20)$. In ovarian cancer, UBQLN1 has been found to be significantly upregulated when exposed to cisplatin (19), indicating that UBQLN1 may mediate cisplatin resistance in ovarian cancer. In lung cancer, both UBQLN1 mRNA $(34,36)$ and protein (36) have been found to be highly expressed in primary lung adenocarcinoma, and higher UBQLN1 levels have been shown to be associated with the shorter survival of patients with lung cancer (34). However, in NSCLC, certain contradictory results have been reported. UBQLN1 has been observed to be absent and underexpressed in $\sim 50 \%$ of tumor tissues (6). The silencing of UBQLN1 in a NSCLC cell line has been shown to increase cell proliferation, migration and invasion, actin cytoskeleton reorganization, and the induction of EMT, leading to a more invasive cell phenotype $(6,11,35)$. 
A

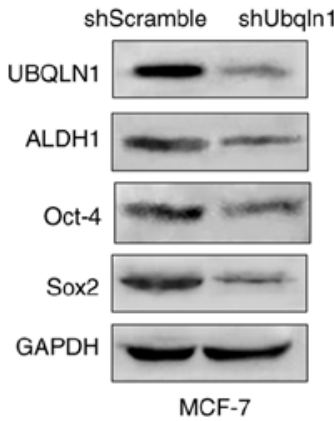

C

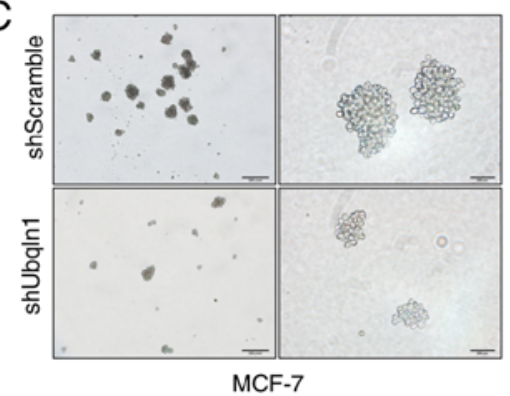

$\mathrm{E}$

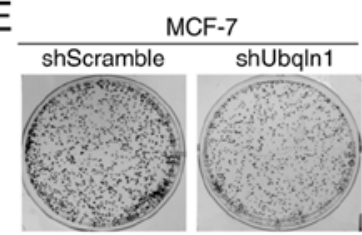

MDA-MB-231

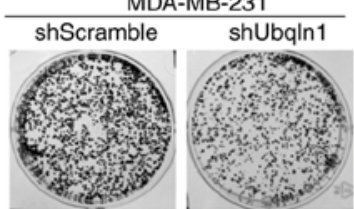

$\mathrm{F}$
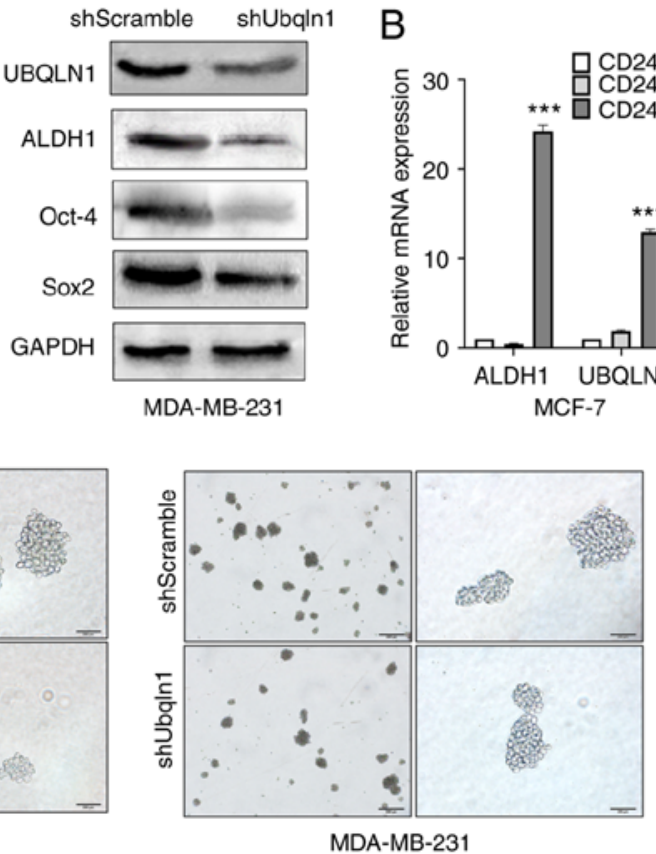

G
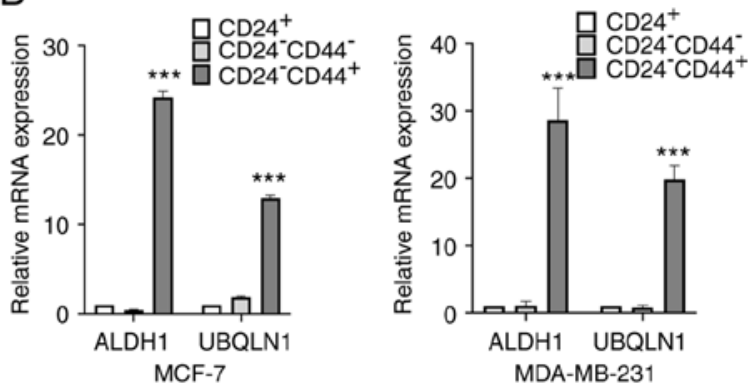

$D$
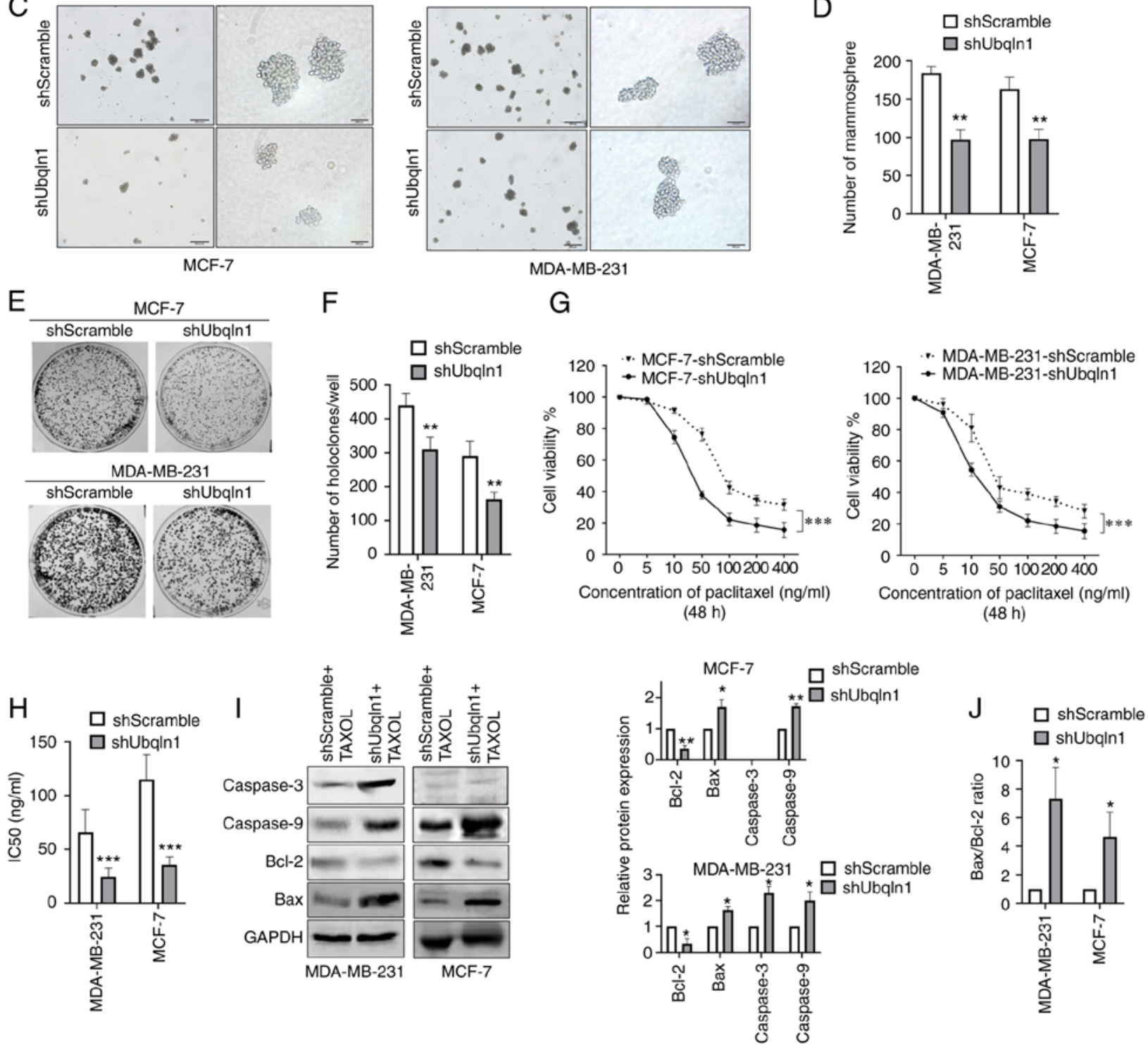

Figure 4. UBQLN1 knockdown attenuates stemness and chemoresistance to paclitaxel in breast cancer. MDA-MB-231 and MCF-7 cells were stably transfected with UBQLN1 shRNA (shUbqln1) or control shRNA (shScramble) and then subjected to different assays. (A) Western blot analysis of UBQLN1, ALDH1, Oct-4 and Sox2. (B) Reverse transcription-quantitative PCR analysis of UBQLN1 and the stem cell marker, ALDH1, in breast cancer cells sorted using magnetic-activated cell sorting system by CD24 or CD44 markers. (C) Mammosphere formation assay and (D) quantification. (E) Holoclone colony formation assay. (F) Histograms indicate mean holoclone numbers formed by 1,000 starting cells. (G) MTT assay was performed to examine cell viability after treating the cells with the indicated dose of paclitaxel for $48 \mathrm{~h}$. (H) Bar graph showing the $\mathrm{IC}_{50}$ values. (I) Cells were exposed to $50 \mathrm{ng} / \mathrm{ml}$ paclitaxel for $48 \mathrm{~h}$, and the levels of Bcl-2, Bax, cleaved caspase-3 and cleaved caspase-9 were then detected by western blotting. Bar graphs illustrate relative protein expression levels. (J) Ratio of Bax to Bcl-2. Data represent means \pm SEM of three independent experiments. ${ }^{*} \mathrm{P}<0.05,{ }^{* *} \mathrm{P}<0.01$ and ${ }^{* * *} \mathrm{P}<0.001$. UBQLN1, ubiquilin-1; shRNA, short hairpin RNA; TAXOL, paclitaxel.

These data raise doubts concerning the biological role of UBQLN1 in cancer, and further research is therefore warranted to clarify the role of UBQLN1 in cancer.
To further elucidate the biological role of UBQLN1 in breast cancer, the influence of UBQLN1 knockdown on biological behavior in vitro was investigated, in particular 

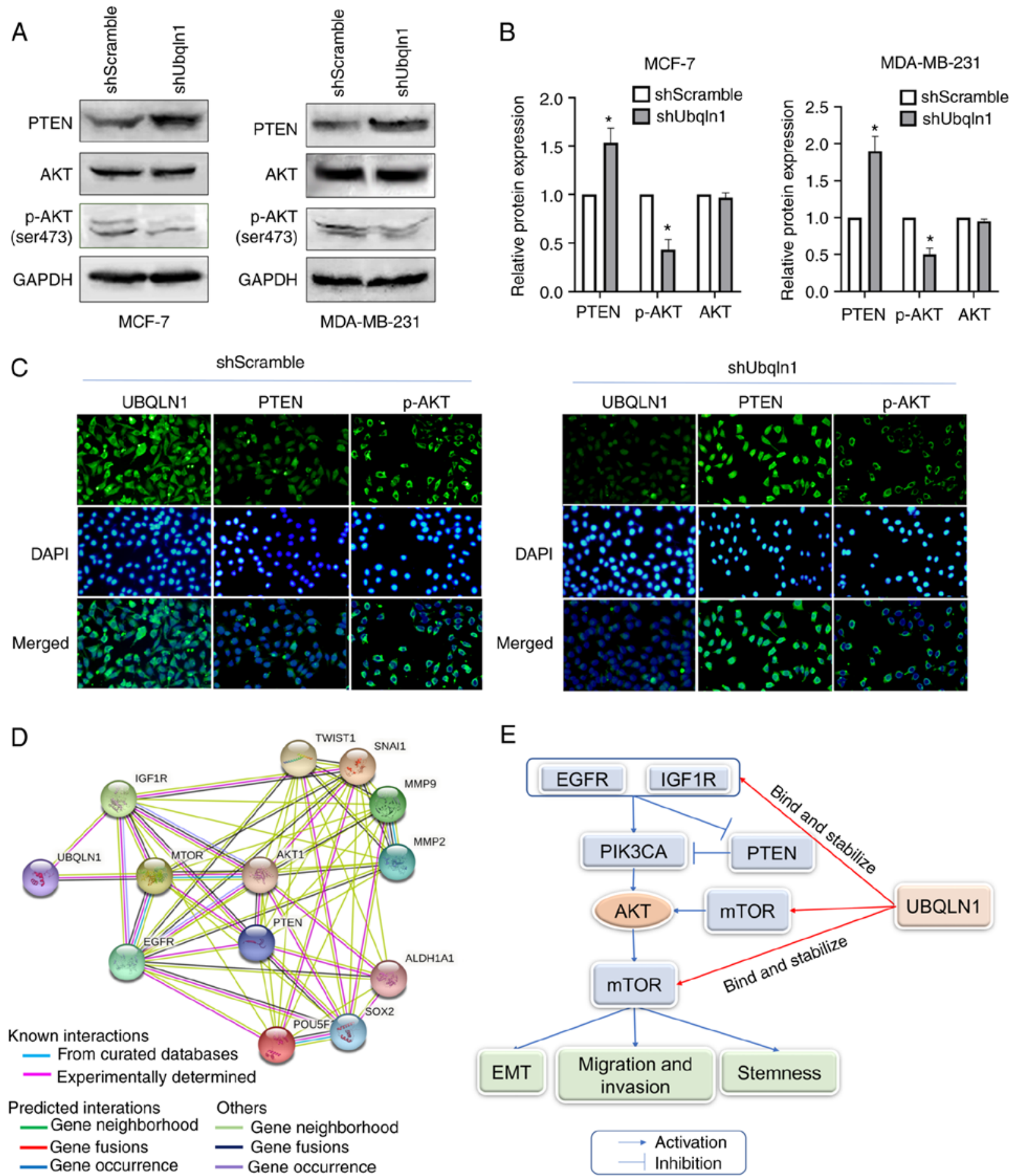

Figure 5. UBQLN1 knockdown inhibits stemness, cell invasion and EMT via the PI3K-AKT signaling pathway in breast cancer. (A) Western blot analysis of the AKT pathway constituents AKT, p-AKT and PTEN. (B) Western blot quantification data. ${ }^{*} \mathrm{P}<0.05$. (C) Representative microphotographs of immunofluorescence staining of UBQLN1, p-AKT and PTEN. (D) Protein-protein interaction network of UBQLN1 and molecules involved in PI3K-AKT signaling according to STRING. (E) Proposed model of the molecular mechanisms through which UBQLN1 promotes EMT and stemness via PI3K-AKT signaling. UBQLN1, ubiquilin-1; EMT, epithelial-to-mesenchymal transition; p-, phosphorylated.

regarding metastasis. In contrast to the tumor suppressor role reported in lung cancer cells $(6,11,35)$, the present study found that the knockdown of UBQLN1 inhibited cell migration and invasion, EMT, and MMP2 and MMP9 expression in breast cancer cell lines. EMT has been implicated in carcinogenesis, and confers metastatic properties to cancer cells by enhancing mobility and invasion (37). MMP2 and MMP9 belong to a group of zinc-containing enzymes that are responsible for the degradation of extracellular matrix components and play pivotal roles in tumor growth and metastasis (30). The secretion and activation of MMPs is a critical step of EMT (38). These data suggested that UBQLN1 is associated with a more migratory and invasive phenotype of breast cancer, and also that UBQLN1 knockdown can effectively inhibit EMT.

It has been reported that BCSCs play a vital role in metastasis and therapeutic resistance (37). The number of 
studies on the association between UBQLN1 and stem cells is limited. In a study on proteomic analysis of a proliferating and differentiating human neuronal stem cell line, the results revealed a significantly decreased UBLQN1 protein expression during stem cell differentiation (21). RNA-sequencing in human embryonic stem cells has demonstrated that UBQLN1 is highly expressed in a cluster of cells with long telomeres and a higher expression of known pluripotency markers (22). These studies indicated an increased level of UBQLN1 expression in stem cells. In the present study, a higher expression of UBQLN1 mRNA in BCSCs was detected, in comparison with that of non-BCSCs. Additionally, UBQLN1 silencing in breast cancer significantly downregulated the expression of the stem cell markers, ALDH1, Oct-4 and Sox2, decreased the mammosphere formation ability and increased the sensitivity of breast cancer cells to paclitaxel therapy. These data thus suggest that UBQLN1 is highly expressed in BCSCs and maintains the stemness properties of breast cancer, including self-renewal and chemoresistance.

The molecular mechanisms underlying the functions of UBQLN1 in the metastasis and stemness of breast cancer are unknown. UBQLN1 was first identified as one of the DAN-binding proteins (DA41) that was expressed at low levels in quiescent cells, and was significantly increased between the $G_{1}$ and $S$ phases of the cell cycle (39). It was also identified as a protein linking IAP with the cytoskeleton (PLIC), which mediates the interaction between integrin-associated protein (IAP) and vimentin-containing intermediate filaments (40). The overexpression of PLICs has been shown to increase IAP-dependent cell spreading and increase the vimentin association with IAP at the plasma membrane (40). UBQLNs have been found to be functionally linked to the UPS and act as ubiquitin receptors (41). UBQLN1 is a component of the protein quality control system, and is important for protein degradation and stabilization (42). UBQLN1 facilitates the proteasome-mediated degradation of certain proteins, including ataxin $3(14,43)$, epidermal growth factor receptor pathway substrate $15(14,43,44)$, Homo sapiens J domain protein 1a (43) and the viral polymerase nonstructural protein 5B (45). For certain ubiquitin-dependent proteasome substrates such as I $\mathrm{B} \alpha$ and P53, overexpression of UBQLN1 interferes with degradation (46). UBQLN1 binds and stabilizes presenilin $1 / 2$ (47), $\gamma$-aminobutyric acid type A (48), BCLb (34), EGFR (11), IGF1R (18), mTOR $(35,49)$ and extended synaptotagmin 2 (14). The mechanism determining the fate of UBQLN1 interacting partners remains unknown.

Among these proteins, EGFR, IGF1R and mTOR are all closely related to AKT signaling, which has been widely reported to participate in tumorigenesis (Fig. 5D) (9), particularly in metastasis and stemness $(7,10)$. EGFR and IGF1R function as oncogenes and promote the development and progression of numerous cancer types $(50,51)$. PI3K/AKT signaling is one of the most critical cancer-promoting pathways through the upregulation of tyrosine kinase receptors $(52)$. Kurlawala et al $(11,18)$ reported that the loss of UBQLN1 led to a marked decrease in total EGFR and IGF1R, particularly when stimulated with their ligands, and the interactions with UBQLN1 stabilized these receptors without affecting the activation of these molecules. However, the effect of this interaction on the activation of downstream signaling was not detected. UBQLN1 can also interact with another molecule involved in PI3K/AKT signaling, namely mTOR $(35,49)$. It has been reported that the loss of UBQLN1 markedly inhibits mTOR phosphorylation and activates autophagy activity (35). Therefore, UBQLN1 may be crucial for maintaining the stability of EGFR, IGF1R and mTOR, thus influencing the activation of PI3K-AKT signaling (Fig. 5E). The UBQLN1-related signaling pathway was estimated by IPA, and the results showed that the canonical pathways of UBQLN1 were involved in the process of neoplastic transformation, including AKT signaling, MYC signaling and cell cycle regulation (34). Although previous evidence has suggested that UBQLN1 may affect the activation of AKT signaling $(34,35,49)$, whether and how UBQLN1 modulates AKT signaling in cancer remains unclear. The present study revealed that UBQLN1 knockdown significantly decreased the expression of p-AKT without affecting total AKT protein levels. In addition, PTEN, a plasma-membrane lipid phosphatase antagonizing the PI3K/AKT pathway (53), demonstrated a significantly increased expression in UBQLN1-depleted breast cancer cells. PPI network analysis by STRING indicated that UBQLN1 and molecules associated with EMT and stemness are all closely correlated with AKT signaling. The present results and those of previous studies suggest that UBQLN1 promotes the migration, invasion, EMT and the stemness of breast cancer by maintaining the activation of AKT signaling, possibly by stabilizing EGFR, IGF1R and mTOR (Fig. 5D and E).

In conclusion, the present study revealed that UBQLN1 expression was upregulated in breast cancer tissues and was associated with a poor prognosis. UBQLN1 knockdown inhibited the migration, invasion, EMT and the stemness of breast cancer cells, and attenuated AKT signaling activation. It was also suggested that UBQLN1 facilitated tumor progression by maintaining the activation of AKT signaling. However, further studies are required to validate the current findings on UBQLN1 function in breast cancer progression by gain-of-function studies and to investigate the specific molecular mechanism of this protein degradation-related protein in the regulation of AKT signaling activation.

\section{Acknowledgements}

Not applicable.

\section{Funding}

The present study was supported by the Dalian Science and Technology Innovation Fund (grant no. 2019J12SN53).

\section{Availability of data and materials}

The datasets used and/or analyzed during the current study are available from the corresponding author on reasonable request.

\section{Authors' contributions}

LL, BS and XY conceived and designed the experiments. XF, TQ, AC, SF and BW performed the experiments. XF, QZ and 
$\mathrm{XY}$ performed the data collection and analyses. XY and XF wrote the original draft. XF, XY and LL confirm the authenticity of the all raw data. All authors have read and approved the final manuscript.

\section{Ethics approval and consent to participate}

The present study and experimental procedures were approved by the Ethics Committee of Dalian Medical University [Dalian, China; (IRB approval no. 2021006)]. Written informed consent was obtained from all patients or patients' families. The study was conducted according to the principles outlined in the Declaration of Helsinki.

\section{Patient consent for publication}

Not applicable.

\section{Competing interests}

The authors declare that they have no competing interests.

\section{References}

1. Sung H, Ferlay J, Siegel RL, Laversanne M, Soerjomataram I, Jemal A and Bray F: Global cancer statistics 2020: GLOBOCAN estimates of incidence and mortality Worldwide for 36 cancers in 185 Countries. CA Cancer J Clin 71: 209-249, 2021.

2. Thorat MA and Balasubramanian R: Breast cancer prevention in high-risk women. Best Pract Res Clin Obstet Gynaecol 65: 18-31, 2020.

3. Nandy SB and Lakshmanaswamy R: Cancer stem cells and metastasis. Prog Mol Biol Transl Sci 151: 137-176, 2017.

4. Najafi M, Farhood B and Mortezaee K: Cancer stem cells (CSCs) in cancer progression and therapy. J Cell Physiol 234: 8381-8395, 2019.

5. Yu X, Zhang F, Mao J, Lu Y, Li J, Ma W, Fan S, Zhang C, Li Q, Wang B, et al: Protein tyrosine phosphatase receptor-type $\delta$ acts as a negative regulator suppressing breast cancer. Oncotarget 8 : 98798-98811, 2017.

6. Shah PP, Lockwood WW, Saurabh K, Kurlawala Z, Shannon SP, Waigel S, Zacharias W and Beverly LJ: Ubiquilin1 represses migration and epithelial-to-mesenchymal transition of human non-small cell lung cancer cells. Oncogene 34: 1709-1717, 2015.

7. Karimi Roshan M, Soltani A, Soleimani A, Rezaie Kahkhaie K, Afshari AR and Soukhtanloo M: Role of AKT and mTOR signaling pathways in the induction of epithelial-mesenchymal transition (EMT) process. Biochimie 165: 229-234, 2019.

8. Gonzalez DM and Medici D: Signaling mechanisms of the epithelial-mesenchymal transition. Sci Signal 7: re8, 2014.

9. Tao C, Luo J, Tang J, Zhou D, Feng S, Qiu Z, Putti TC, Xiang T, Tao Q, Li L and Ren G: The tumor suppressor Zinc finger protein 471 suppresses breast cancer growth and metastasis through inhibiting AKT and $\mathrm{Wnt} / \beta$-catenin signaling. Clin Epigenetics 12: 173, 2020.

10. Xia $\mathrm{P}$ and $\mathrm{Xu} \mathrm{XY}$ : $\mathrm{PI} 3 \mathrm{~K} / \mathrm{Akt} / \mathrm{mTOR}$ signaling pathway in cancer stem cells: From basic research to clinical application. Am J Cancer Res 5: 1602-1609, 2015.

11. Kurlawala Z, Saurabh K, Dunaway R, Shah PP, Siskind LJ and Beverly LJ: Ubiquilin proteins regulate EGFR levels and activity in lung adenocarcinoma cells. J Cell Biochem 122: 43-52, 2021.

12. Bao J, Jiang X, Zhu X, Dai G, Dou R, Liu X, Sheng H, Liang Z and $\mathrm{Yu} \mathrm{H}$ : Clinical significance of ubiquilin 1 in gastric cancer. Medicine(Baltimore) 97: e9701, 2018.

13. Jantrapirom S, Piccolo LL, Pruksakorn D, Potikanond S and Nimlamool W: Ubiquilin networking in cancers. Cancers (Basel) 12: 1586, 2020.

14. Kurlawala Z, Shah PP, Shah C and Beverly LJ: The STI and UBA Domains of UBQLN1 are critical determinants of substrate interaction and proteostasis. J Cell Biochem 118: 2261-2270, 2017.
15. Li X, Zhou J, Chen H, Wang F, Mei Q and Sun H: The association between the UBQLN1 polymorphism and Alzheimer's disease risk: A systematic review. Cell Mol Biol (Noisy-le-grand) 63: 94-96, 2017.

16. Kim SH, Shi Y, Hanson KA, Williams LM, Sakasai R, Bowler MJ and Tibbetts RS: Potentiation of amyotrophic lateral sclerosis (ALS)-associated TDP-43 aggregation by the proteasome-targeting factor, ubiquilin 1. J Biol Chem 284: 8083-8092, 2009.

17. Safren N, El Ayadi A, Chang L, Terrillion CE, Gould TD, Boehning DF and Monteiro MJ: Ubiquilin-1 overexpression increases the lifespan and delays accumulation of Huntingtin aggregates in the R6/2 mouse model of Huntington's disease. PLoS One 9: e87513, 2014.

18. Kurlawala Z, Dunaway R, Shah PP, Gosney JA, Siskind LJ, Ceresa BP and Beverly LJ: Regulation of insulin-like growth factor receptors by Ubiquilin1. Biochem J 474: 4105-4118, 2017.

19. Guidi F, Puglia M, Gabbiani C, Landini I, Gamberi T, Fregona D, Cinellu MA, Nobili S, Mini E, Bini L, et al: 2D-DIGE analysis of ovarian cancer cell responses to cytotoxic gold compounds. Mol Biosyst 8: 985-993, 2012.

20. Wang Y, Lu J, Zhao X, Feng Y, Lv S, Mu Y, Wang D, Fu H, Chen Y and Li Y: Prognostic significance of Ubiquilin1 expression in invasive breast cancer. Cancer Biomark 15: 635-643, 2015.

21. Hoffrogge R, Mikkat S, Scharf C, Beyer S, Christoph H, Pahnke J, Mix E, Berth M, Uhrmacher A, Zubrzycki IZ, et al: 2-DE proteome analysis of a proliferating and differentiating human neuronal stem cell line (ReNcell VM). Proteomics 6: $1833-1847,2006$

22. Wang H, Zhang K, Liu Y, Fu Y, Gao S, Gong P, Wang H, Zhou Z, Zeng M, Wu Z, et al: Telomere heterogeneity linked to metabolism and pluripotency state revealed by simultaneous analysis of telomere length and RNA-seq in the same human embryonic stem cell. BMC Biol 15: 114, 2017.

23. Livak KJ and Schmittgen TD: Analysis of relative gene expression data using real-time quantitative PCR and the 2(-Delta Delta C(T)) Method. Methods 25: 402-408, 2001.

24. Zhang F, Wang B, Qin T, Wang L, Zhang Q, Lu Y, Song B, Yu X and Li L: IL-6 induces tumor suppressor protein tyrosine phosphatase receptor type D by inhibiting miR-34a to prevent IL-6 signaling overactivation. Mol Cell Biochem 473: 1-13, 2020.

25. Qin T, Li B, Feng X, Fan S, Liu L, Liu D, Mao J, Lu Y, Yang J, Yu X, et al: Abnormally elevated USP37 expression in breast cancer stem cells regulates stemness, epithelial-mesenchymal transition and cisplatin sensitivity. J Exp Clin Cancer Res 37: 287, 2018

26. Bartha Á and Győrffy B: TNMplot.com: A web tool for the comparison of gene expression in normal, tumor and metastatic tissues. Int J Mol Sci 22: 2622, 2021.

27. Györffy B, Lanczky A, Eklund AC, Denkert C, Budczies J, Li Q and Szallasi Z: An online survival analysis tool to rapidly assess the effect of 22,277 genes on breast cancer prognosis using microarray data of 1,809 patients. Breast Cancer Res Treat 123: 725-731, 2010.

28. Tang W, Zhou M, Dorsey TH, Prieto DA, Wang XW, Ruppin E, Veenstra TD and Ambs S: Integrated proteotranscriptomics of breast cancer reveals globally increased protein-mRNA concordance associated with subtypes and survival. Genome Med 10: 94, 2018.

29. Jia D, Park JH, Kaur H, Jung KH, Yang S, Tripathi S, Galbraith M, Deng Y, Jolly MK, Kaipparettu BA, et al: Towards decoding the coupled decision-making of metabolism and epithelial-to-mesenchymal transition in cancer. Br J Cancer 124: 1902-1911, 2021.

30. Fu Y, Shao ZM, He QZ, Jiang BQ, Wu Y and Zhuang ZG: Hsa-miR-206 represses the proliferation and invasion of breast cancer cells by targeting Cx43. Eur Rev Med Pharmacol Sci 19: 2091-2104, 2015

31. Yu JM, Sun W, Wang ZH, Liang X, Hua F, Li K, Lv XX, Zhang XW, Liu YY, Yu JJ, et al: TRIB3 supports breast cancer stemness by suppressing FOXO1 degradation and enhancing SOX2 transcription. Nat Commun 10: 5720, 2019.

32. Abu Samaan TM, Samec M, Liskova A, Kubatka P and Büsselberg D: Paclitaxel's mechanistic and clinical effects on breast cancer. Biomolecules 9: 789, 2019.

33. Jänicke RU: MCF-7 breast carcinoma cells do not express caspase-3. Breast Cancer Res Treat 117: 219-221, 2009.

34. Beverly LJ, Lockwood WW, Shah PP, Erdjument-Bromage H and Varmus $\mathrm{H}$ : Ubiquitination, localization, and stability of an anti-apoptotic BCL2-like protein, BCL2L10/BCLb, are regulated by Ubiquilin1. Proc Natl Acad Sci USA 109: E119-E126, 2012. 
35. Zhang $\mathrm{X}, \mathrm{Su} \mathrm{Y}$, Lin $\mathrm{H}$ and Yao X: The impacts of ubiquilin 1 (UBQLN1) knockdown on cells viability, proliferation, and apoptosis are mediated by p53 in A549 lung cancer cells. J Thorac Dis 12: 5887-5895, 2020.

36. Chen G, Wang X, Yu J, Varambally S, Yu J, Thomas DG, Lin MY, Vishnu P, Wang Z, Wang R, et al: Autoantibody profiles reveal ubiquilin 1 as a humoral immune response target in lung adenocarcinoma. Cancer Res 67: 3461-3467, 2007.

37. Mittal V: Epithelial mesenchymal transition in tumor metastasis Annu Rev Pathol 13: 395-412, 2018.

38. Agraval $\mathrm{H}$ and Yadav UCS: MMP-2 and MMP-9 mediate cigarette smoke extract-induced epithelial-mesenchymal transition in airway epithelial cells via EGFR/Akt/GSK3 $\beta / \beta$-catenin pathway: Amelioration by fisetin. Chem Biol Interact 314: 108846,2019

39. Hanaoka E, Ozaki T, Ohira M, Nakamura Y, Suzuki M, Takahashi E, Moriya H, Nakagawara A and Sakiyama S: Molecular cloning and expression analysis of the human DA41 gene and its mapping to chromosome 9q21.2-q21.3. J Hum Genet 45: 188-191, 2000.

40. Wu AL, Wang J, Zheleznyak A and Brown EJ: Ubiquitin-related proteins regulate interaction of vimentin intermediate filaments with the plasma membrane. Mol Cell 4: 619-625, 1999.

41. Gadhave K, Kumar P, Kapuganti SK, Uversky VN and Giri R: Unstructured biology of proteins from ubiquitin-proteasome system: Roles in cancer and neurodegenerative diseases. Biomolecules 10: 796, 2020.

42. Zhang C and Saunders AJ: An emerging role for Ubiquilin 1 in regulating protein quality control system and in disease pathogenesis. Discov Med 8: 18-22, 2009.

43. Heir R, Ablasou C, Dumontier E, Elliott M, Fagotto-Kaufmann C and Bedford FK: The UBL domain of PLIC-1 regulates aggresome formation. EMBO Rep 7: 1252-1258, 2006.

44. Regan-Klapisz E, Sorokina I, Voortman J, de Keizer P, Roovers RC, Verheesen P, Urbé S, Fallon L, Fon EA, Verkleij A, et al: Ubiquilin recruits Eps15 into ubiquitin-rich cytoplasmic aggregates via a UIM-UBL interaction. J Cell Sci 118(Pt 19): 4437-4450, 2005.
45. Gao L, Tu H, Shi ST, Lee KJ, Asanaka M, Hwang SB and Lai MM: Interaction with a ubiquitin-like protein enhances the ubiquitination and degradation of hepatitis $\mathrm{C}$ virus RNA-dependent RNA polymerase. J Virol 77: 4149-4159, 2003.

46. Kleijnen MF, Shih AH, Zhou P, Kumar S, Soccio RE, Kedersha NL, Gill G and Howley PM: The hPLIC proteins may provide a link between the ubiquitination machinery and the proteasome. Mol Cell 6: 409-419, 2000.

47. Mah AL, Perry G, Smith MA and Monteiro MJ: Identification of ubiquilin, a novel presenilin interactor that increases presenilin protein accumulation. J Cell Biol 151: 847-862, 2000.

48. Bedford FK, Kittler JT, Muller E, Thomas P, Uren JM, Merlo D, Wisden W, Triller A, Smart TG and Moss SJ: GABA(A) receptor cell surface number and subunit stability are regulated by the ubiquitin-like protein Plic-1. Nat Neurosci 4: 908-916, 2001.

49. Wu S, Mikhailov A, Kallo-Hosein H, Hara K, Yonezawa K and Avruch J: Characterization of ubiquilin 1, an mTOR-interacting protein. Biochim Biophys Acta 1542: 41-56, 2002.

50. Cao J and Yee D: Disrupting insulin and IGF receptor function in cancer. Int J Mol Sci 22: 555, 2021.

51. Santos ED, Nogueira KA, Fernandes LC, Martins JR, Reis AV, Neto JB, Júnior IJ, Pessoa C, Petrilli R and Eloy JO: EGFR targeting for cancer therapy: Pharmacology and immunoconjugates with drugs and nanoparticles. Int J Pharm 592: 120082, 2021.

52. Gallardo A, Lerma E, Escuin D, Tibau A, Muñoz J, Ojeda B, Barnadas A, Adrover E, Sánchez-Tejada L, Giner D, et al: Increased signalling of EGFR and IGF1R, and deregulation of PTEN/PI3K/Akt pathway are related with trastuzumab resistance in HER2 breast carcinomas. Br J Cancer 106: 1367-1373, 2012.

53. Trotman LC, Wang X, Alimonti A, Chen Z, Teruya-Feldstein J, Yang H, Pavletich NP, Carver BS, Cordon-Cardo C, ErdjumentBromage $\mathrm{H}$, et al: Ubiquitination regulates PTEN nuclear import and tumor suppression. Cell 128: 141-156, 2007.

This work is licensed under a Creative Commons Attribution-NonCommercial-NoDerivatives 4.0 International (CC BY-NC-ND 4.0) License. 